\title{
Numbers of spermatozoa required to effect a normal rate of conception in naturally mated Merino ewes
}

\author{
W. J. Fulkerson*, Anthea L. Synnott and D. R. Lindsay \\ Department of Animal Science and Production, University of Western A ustralia, Nedlands, \\ Western A ustralia 6009, A ustralia
}

\begin{abstract}
Summary. The semen deposited by rams into ewes at a single insemination was estimated from the semen samples collected into intravaginal devices before and after the rams had been mated to oestrous ewes. Of 109 naturally cyclic ewes receiving $>60 \times 10^{6}$ spermatozoa (from $5 \mathrm{rams}$ with ejaculates containing $<10 \times 10^{6}$ $1000 \times 10^{6}$ spermatozoa $) 95 \%$ became pregnant compared with $31 \%(P<0.001)$ of ewes receiving $<60 \times 10^{6}$ spermatozoa. It is concluded that the number of spermatozoa deposited at a single insemination by continuously mated rams may be insufficient to ensure good fertility, and that more than one insemination may be necessary.
\end{abstract}

\section{Introduction}

In artificial insemination (A.I.), for which the aim is to utilize spermatozoa as sparingly as possible, a minimum dose of $120-125 \times 10^{6}$ spermatozoa per insemination will lead to a normal rate of conception (Salamon, 1962; Allison \& Robinson, 1971). No equivalent minimum dose for natural insemination has been proposed because it is difficult to measure the numbers of spermatozoa which a ram ejaculates into the vagina of the ewe. It might be assumed that the minimum dose for optimum fertility is higher than for A.I. because rams are presumably less accurate than inseminators in placing semen in the mouth of the cervix.

In this experiment we have devised a method of estimating the number of spermatozoa received by naturally mated ewes and have used this to estimate the minimum number necessary to ensure a reasonable chance of conception.

\section{Materials and Methods}

The experiment was carried out during the spontaneous breeding season of Merino ewes (February to March in 1979 and 1980) in the south of Western Australia.

\section{Animals}

Two hundred (200) intact 4-year-old and 120 ovariectomized Merino ewes together with 6 vasectomized (teasers) and 5 intact mature Merino rams, were used. All animals were fed ad libitum a ration of lupin grain and meadow hay throughout the experiment.

* Present address: Department of Agriculture, 2 George Street, Launceston, Tasmania 7250, Australia. 


\section{Experimental procedure}

Depletion of sperm reserves in intact rams. Oestrus was induced in ovariectomized ewes by injecting them subcutaneously every 2 days for 14 days with $20 \mathrm{mg}$ progesterone (Calbiochem, California, U.S.A.) and then $48 \mathrm{~h}$ later with $60 \mu \mathrm{g}$ oestradiol benzoate (Intervet, Oss, The Netherlands) (Robinson, Moore \& Binet, 1956). Each intact ram was enclosed separately with 4 fresh oestrous (induced) ewes each day for different numbers of days. The longer the ram remained and mated with oestrous ewes the further his reserves of spermatozoa were depleted. We were thus able to select, within limits, rams with predictable numbers of spermatozoa per ejaculate.

Detection of oestrus in, and mating of, intact ewes. The intact ewes were run continuously with teaser rams fitted with 'Sire-Sine' harnesses and marking crayons (Radford, Watson \& Wood, 1960). The teasers were fitted with cloth aprons to prevent intromission and insemination of the ewes.

At 08:00 and 17:00 h each day, ewes coming into oestrus in the previous 9-15 h (identified by crayon marks on the backs) were removed from the flock. A ram was selected and the ram and a ewe were left alone in the mating pen until mating was completed. Each ewe received only one ejaculation at the mating.

Determination of the number of spermatozoa inseminated. At $30 \mathrm{~min}$ before and $30 \mathrm{~min}$ after each intact ewe was inseminated by an intact ram, the ram was allowed to ejaculate into an ovariectomized ewe in which oestrus was induced as previously described and which was fitted with an intravaginal semen collector device (Synnott, Fulkerson \& Lindsay, 1981). The numbers of spermatozoa ejaculated into the 2 ovariectomized ewes were plotted against time and a line drawn between the two points. From this relationship and from a knowledge of the time at which the intact ewes were mated, the number of spermatozoa deposited into each intact ewe was estimated.

Pregnancy diagnosis. Pregnancy was determined at 8 weeks after mating by recording returns to service up to that time, and then verified by laparoscopy.

Analysis of semen. The semen collection device was removed from the ewe and the spermatozoa were thoroughly washed with $400 \mathrm{ml}$ saline $(9 \mathrm{~g} \mathrm{NaCl} / 1)$ plus $0.1 \%(\mathrm{v} / \mathrm{v})$ of $40 \%$ formaldehyde. The number of spermatozoa was counted on a Coulter Counter using the method of Iversen (1963). The ratio of live to dead spermatozoa was estimated for each ram and the final estimate of the number of live spermatozoa deposited by the ram was calculated from this ratio and the known rate of recovery of spermatozoa from the semen collection device (Synnott et al., 1981).

\section{Results}

A total of 109 ewes was inseminated by rams. Ewes were grouped according to the estimated numbers of live spermatozoa they received at mating. Text-figure 1 shows that $95 \%(39 / 42)$ of ewes became pregnant when they received more than $60 \times 10^{6}$ spermatozoa in a single ejaculate at that mating. The rate of pregnancy in ewes receiving $50 \times 10^{6}$ spermatozoa or fewer was significantly lower $\left(31 \% ; P<0.001 \chi^{2}\right.$ test) and varied between 9 and $47 \%$ per class group.

\section{Discussion}

Contrary to expectation only $60 \times 10^{6}$ spermatozoa from a single natural service were necessary for good conception under the conditions of this experiment. By contrast, a single artificial insemination requires twice as many spermatozoa for equivalent conception (Salamon, 1962; 


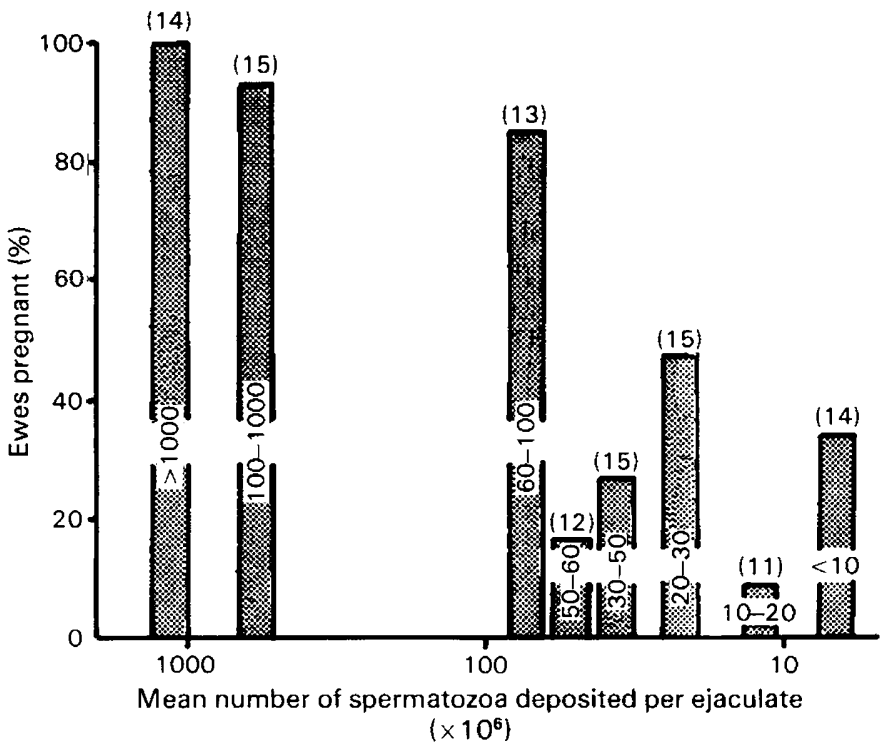

Text-fig. 1. Percentage of ewes pregnant in relation to the estimated number of spermatozoa received from a single ejaculate at mating. The number of animals contributing to each group is indicated in parentheses.

Allison \& Robinson, 1971). Assuming that semen of equivalent quality is used, the only consistent differences between natural and artificial insemination appear to be the absence of, or interruption to, normal courtship behaviour of the ewe and the stress associated with handling and strange surroundings when A.I. is used. If these factors are as important as our results suggest, a review of A.I. techniques to account for them would be worthwhile. Restall (1961) has already shown that A.I. was more successful when ewes were returned to vasectomized rams immediately after insemination than when they were isolated from rams. On the other hand a large farmer-based organization in Western Australia has claimed success for a technique in which semen is deposited by pipette blindly into the vagina (J. H. Shepherd, personal communication). It seems possible that the absence of obvious stress to the ewes in this technique may be compensating for the haphazard placement of semen.

In natural service, rams often deposited fewer spermatozoa per service (Allison, 1972; Synnott $e t$ al., 1981) than we have shown here to be adequate for good conception. Indeed, some of the rams in this study produced less than one-fifth of an adequate dose after continual mating. Therefore, observations in the field that the chances of a ewe conceiving are related to the number of times she is served (Mattner \& Braden, 1967) or the more rams with which she mates (Knight \& Lindsay, 1973) can be explained on the basis of inadequate numbers of spermatozoa in a single insemination.

We thank Mr T. Boyes and Mr R. Armstrong for providing skilled technical assistance. One of us (W. J. F.) was the E. H. B. Lefroy Fellow during the course of the experiment. Financial assistance was provided by a grant from the Australian Meat Board.

\section{References}

Allison, A.J. (1972) The effect of mating pressure on characteristics of the ejaculate in rams and on reproductive performance in ewes. Proc. N.Z. Soc. Anim. Prod. 32, 112-113.
Allison, A. J. \& Robinson, T.J. (1971) Fertility of progesterone-treated ewes in relation to the numbers and concentration of spermatozoa in the inseminate. Aust.J. biol. Sci. 24, 1001-1008. 
Iversen, S. (1963) Evaluation of the number of spermatozoa in bull semen. A comparison between electronic counting, light scattering and absorptiometry. J. agric. Sci., Camb. 62, 219-223.

Knight, T.W. \& Lindsay, D.R. (1973) Identifying the mating performance of individual rams in field flocks. A ust. J. agric. Res. 24, 579-585.

Mattner, P.E. \& Braden, A.W.H. (1967) Studies in flock mating of sheep. 2. Fertilization and prenatal mortality. Aust. J. exp. Agric. Anim. Husb. 7, $110-116$.

Radford, H.M., Watson, R.H. \& Wood, G.F. (1960) A crayon and associated harness for the detection of mating under field conditions. Aust. vet.J. 36, 57-66.

Restall, B.J. (1961) Artificial insemination of sheep. VI. The effect of post inseminal coitus on percentage of ewes lambing to a single insemination. Aust. vet. J. 37, 70-72.

Robinson, T.J., Moore, N.W. \& Binet, F.E. (1956) The effect of the duration of progesterone pre-treatment on the response of the spayed ewe to oestrogen. $J$. Endocr. 14, 1-7.

Salamon, S. (1962) Studies on the artificial insemination of Merino sheep. III. The effect of frequent ejaculation on semen characteristics and fertilizing capacity. Aust. J. agric. Res. 13, 1137-1150.

Synnott, A.L., Fulkerson, W.J. \& Lindsay, D.R. (1981) Sperm output by rams and distribution amongst ewes under conditions of natural mating. J. Reprod. Fert. 61, 355-361.

Received 6 November 1981 